02

\title{
Кинетика генерации неравновесных носителей заряда в кристаллах с глубокими примесями при участии двухцентровых переходов между зонными и примесными состояниями
}

\author{
(C) А.А. Попов, Е.Ю. Перлин, А.В. Иванов \\ Университет ИТМО, Центр „Информационные оптические технологии“, \\ 199034 Санкт-Петербург, Россия \\ e-mail: aapopov@corp.ifmo.ru
}

Поступила в редакцию 15.12.2017 г.

Предложен новый эффективный механизм нелинейного фотовозбуждения прозрачного кристалла с глубокими примесными центрами. Предполагается, что энергия светового кванта меньше энергетического зазора между дном зоны проводимости и примесным уровнем, но больше зазора между примесным уровнем и потолком валентной зоны. Рассмотрена кинетика нелинейной каскадной генерации неравновесных носителей заряда с учетом двухцентровых процессов, в которых передача энергии и поглощение фотона происходят в одном элементарном акте. Получены зависимости концентраций неравновесных носителей заряда в зонах и заполнения примесных состояний от интенсивности лазерного излучения. Показано, что процесс генерации неравновесных электронно-дырочных пар носит пороговый характер. В зависимости от концентрации примесных центров пороговые интенсивности могут составлять $\sim 10^{5}-10^{7} \mathrm{~W} / \mathrm{cm}^{2}$, а время установления квазиравновесных населенностей электронных состояний 10-0.1 ns.

DOI: $10.21883 /$ OS.2018.04.45749.288-17

\section{Введение}

Процессы типа фотонной лавины (ФЛ) интенсивно исследуются в течение последних десятилетий (см., например, [1-20]). К числу основных особенностей ФЛ относится наличие области интенсивностей $j$ возбуждающего ФЛ лазерного излучения, в которой заселенности электронных состояний и оптическое поглощение резко меняются даже при небольшом изменении $j$. В принципе это явление можно было бы использовать для реализации оптических переключений, однако бо́льшая часть работ по эффекту ФЛ выполнена для систем примесных редкоземельных ионов. В этих системах в силу малости сил осцилляторов актуальных оптических переходов и сравнительно больших времен продольной релаксации примесных электронов процессы переключения оказываются чрезвычайно медленными, с характерными временами порядка миллисекунд. Более быстрые переключения возможны в гетероструктурах с глубокими квантовыми ямами либо в объемных кристаллах с весьма специфической зонной структурой, которой обладают сравнительно немногие материалы [21-32]. Рассмотренные в [21-32] оптические переключения могут быть реализованы как при очень высоких интенсивностях лазерного излучения $\left(j \sim 10^{11}-10^{13} \mathrm{~W} / \mathrm{cm}^{2}\right)$, когда такое переключение может привести к пробою материала [24,29], так и при умеренных интенсивностях [21-23,25-28,30-32], когда переключения данного типа могут быть использованы в быстродействующих оптических затворах и лимитерах. В настоящей работе показывается, что меха- низм, аналогичный тому, что был рассмотрен в работах [30-32] для случая кристаллов со специальными типами электронной зонной структуры, может быть реализован и в материалах с вполне стандартной зонной структурой при наличии глубоких примесных центров.

В работах [33,34] были рассчитаны вероятности двухэлектронных переходов между зонными и примесными состояниями в ситуации, когда на кристалл подается лазерное излучение с энергией кванта $\hbar \omega$, меньшей, чем энергетические зазоры между зонными и примесными состояниями. В случае перехода „валентная зона-примесь“ дефицит энергии, необходимый для перехода, покрывается за счет передачи энергии, высвобождающейся при переходе электрона из зоны проводимости на уровень соседнего примесного центра. В случае перехода „примесь-зона проводимости“ электрон в зоне проводимости, совершающий внутризонный переход из состояния с большой кинетической энергией в состояние с меньшей кинетической энергией, передает энергию на покрытие дефицита. В настоящей работе предлагается модель генерации неравновесных электронно-дырочных пар (ЭДП) в кристаллах с глубокими примесями на основе первого из этих процессов.

\section{Модель нелинейной генерации неравновесных носителей заряда}

Рассмотрим кристалл (рис. 1) с шириной прямой запрещенной зоны $E_{g}$. Предположим, что в кристалле 


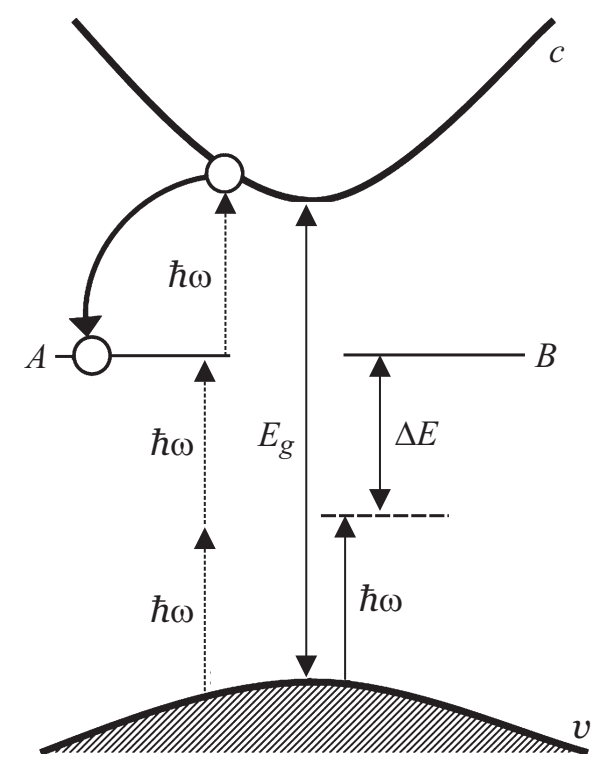

Рис. 1. Схема генерации неравновесных ЭДП. Пунктирные линии со стрелками - каскад двухфотонно-однофотонных переходов. Сплошные линии со стрелками - двухцентровый переход свободных носителей заряда.

имеется достаточно высокая концентрация глубоких примесных центров. Пусть на кристалл действует лазерное излучение с энергией кванта $\hbar \omega$. Кроме того, предположим, что энергетический зазор $\left|E_{t}\right|$ между дном зоны проводимости $c$ и примесным уровнем составляет величину, меньшую $\hbar \omega$, а энергетический зазор $E_{g}-\left|E_{t}\right|$ между примесным состоянием и потолком валентной зоны $v$ больше $\hbar \omega$, но меньше $2 \hbar \omega$. Если до начала действия светового импульса свободные носители заряда (электроны $e$ в зоне проводимости $c$ и дырки $h$ в валентной зоне $v$ ) отсутствуют, они могут появиться в результате каскадного процесса, состоящего из двухфотонного перехода между валентной зоной $v$, где образуются дырки $h$, и примесным уровнем, а также однофотонного перехода между примесным уровнем и зоной проводимости $c$. Попавшие в зону $c$ электроны за счет процессов внутризонной релаксации достаточно быстро (за времена $\sim 10^{-13} \mathrm{~s}$ ) опускаются в область дна зоны проводимости, откуда они могут захватываться на примесные центры, либо рекомбинировать с дырками, которые аналогичным образом попадают в область потолка зоны $v$. В то же время свободные электроны могут участвовать в двухэлектронном процессе $e+\hbar \omega \rightarrow A, B, h$ : при поглощении фотона $\hbar \omega$ образуется пара, состоящая из электрона на центре $B$ и дырки $h$ в валентной зоне $v$, а дефицит энергии покрывается за счет перехода электрона $e$ из зоны проводимости $c$ на примесный центр A. Затем электроны с каждого из центров переходят, поглощая по фотону $\hbar \omega$, в зону проводимости. В результате повторения таких процессов при определенных условиях происходит лавинообразное нарастание концентраций свободных электронов и дырок, а также заполнение примесных состояний.

\section{Кинетика фотопереходов}

При не очень большой интенсивности света $\left(j \sim 10^{8} \mathrm{~W} / \mathrm{cm}^{2}\right)$ период осцилляций Раби для переходов между зонными и примесными состояниями и длительность светового импульса значительно больше времен внутризонной релаксации возбужденных носителей. В этой ситуации для описания кинетики фотопереходов можно использовать уравнения баланса для концентраций $n_{c}$ и $n_{\lambda}$ в зоне проводимости $c$ и в состоянии примесного центра $t$. Эти уравнения имеют следующий вид:

$$
\begin{aligned}
\dot{n}_{c}(t)= & \sigma_{t c}^{(1)} j n_{t}(t) n_{i} \\
& -\gamma_{v t}^{(1)} j n_{c}(t)\left[1-n_{t}(t)\right]^{2} n_{i}^{2}-d n_{c}(t) p(t), \\
\dot{n}_{t}(t)= & \sigma_{v t}^{(2)} j^{2}\left[1-n_{t}(t)\right] \\
& +2 \gamma_{v t}^{(1)} j n_{c}(t)\left[\left(1-n_{t}(t)\right)\right]^{2} n_{i}-\sigma_{t c}^{(1)} j n_{t}(t), \\
& p(t)=n_{c}(t)+n_{t}(t) n_{i},
\end{aligned}
$$

где $n_{c}$ и $p$ - концентрации электронов в зоне проводимости и дырок в валентной зоне соответственно, $d$ - скорость бимолекулярной рекомбинации, $n_{i}-$ концентрация примесных центров, $n_{t}$ - заселенность примесных центров, $\sigma_{v t}^{(2)} j^{2}-$ скорость двухфотонного перехода „валентная зона-примесь“, $\sigma_{t c}^{(1)} j-$ скорость однофотонного перехода „примесь-зона проводимости“. $\gamma_{v t}^{(1)} j n_{c}(t)\left[\left(1-n_{t}(t)\right)\right] n_{i}-$ скорости двухцентровых процессов с поглощением фотона. Система решалась с начальными условиями: $p(0)=0, n_{c}(0)=0, n_{t}(0)=0$. Скорость однофотонного перехода „примесь-зона проводимости“ $\sigma_{t c}^{(1)}$ может быть легко получена в модели потенциала нулевого радиуса [35,36]:

$$
\begin{aligned}
\sigma_{t c}^{(1)}= & \frac{2^{7 / 2} e^{2} N_{t}^{2} m_{c}^{3 / 2} \Delta^{1 / 2}}{m^{2} c \sqrt{\varepsilon_{\infty}} \hbar^{4} \omega^{2}} \\
& \times\left[\frac{p_{c v}^{2}}{\left(-E_{g}-\gamma \Delta+\left|E_{t}\right|\right)^{2}}+\frac{2 m^{2} \Delta}{2 m_{c} \hbar^{2} \omega^{2}}\right],
\end{aligned}
$$

где $e-$ заряд электрона, $m$ - масса свободного электрона, $m_{v}$ и $m_{c}$ - эффективные массы дырки в валентной зоне и электрона в зоне проводимости соответственно, $p_{c v}$ - межзонный матричный элемент оператора импульса, $\varepsilon_{\infty}-$ высокочастотная диэлектрическая проницаемость, $E_{t}$ - энергия примесного уровня, отсчитанная от дна зоны проводимости, $\Delta=\hbar \omega-\left|E_{t}\right|$, $\gamma=m_{c} / m_{v}$, коэффициент $N_{t}$ вычисляется по следующей формуле [36]:

$$
N_{t}^{2}=2^{3 / 2} \pi \hbar^{3}\left[m_{c}^{3 / 2} /\left|E_{t}\right|^{1 / 2}+m_{\nu}^{3 / 2} /\left(E_{g}+\left|E_{t}\right|\right)^{1 / 2}\right]^{-1} .
$$


$a$
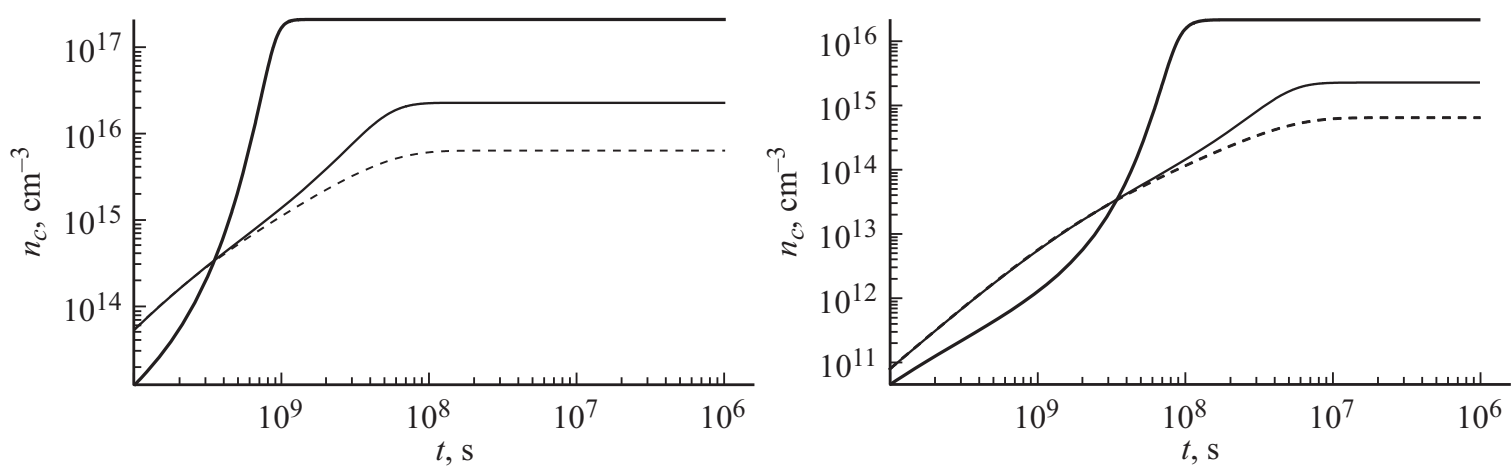

$c$
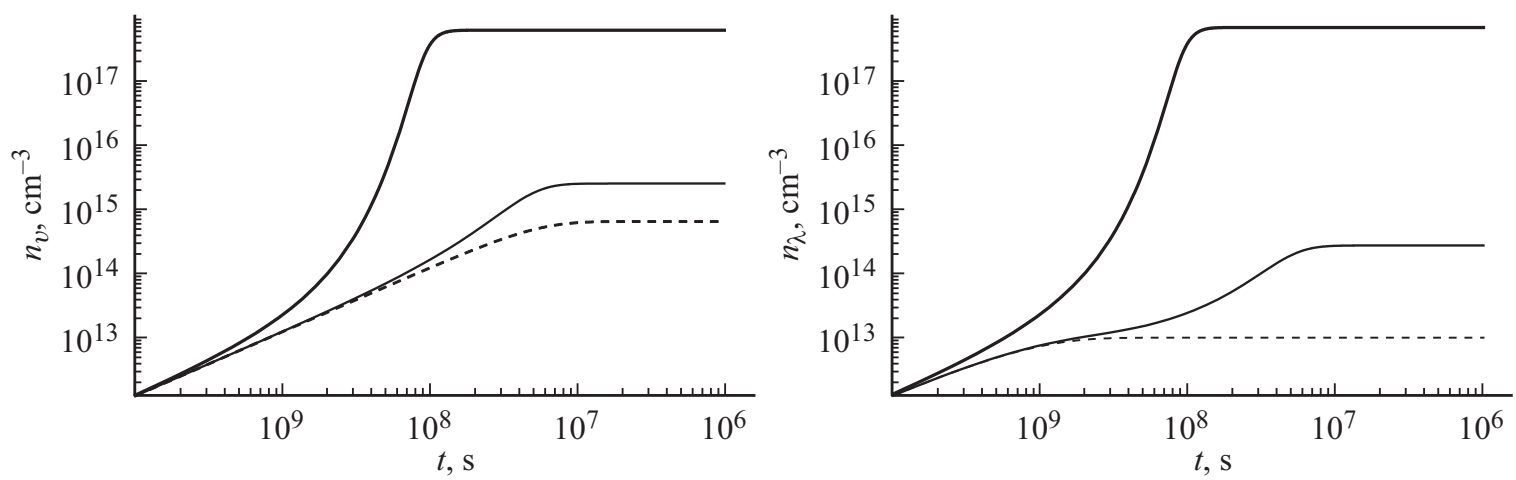

Рис. 2. Зависимости концентрации электронов в зоне проводимости $n_{c}(a, b)$, концентрации неравновесных дырок $n_{v}(c)$ и заселенности примесных состояний $n_{\lambda}=n_{t} \times n_{i}(d)$ от времени действия лазерного излучения. Штриховая линия: в генерации ЭДП участвует только двухфотонно-однофотонный каскад; тонкая сплошная линия: в генерации ЭДП участвует и двухцентровый механизм; жирная сплошная линия: учитывается возможность примесей собираться в кластеры. Средняя по объему кристалла концентрация примесных центров $n_{i}=10^{19} \mathrm{~cm}^{-3}$. Интенсивность излучения $j=10^{6} \mathrm{~W} / \mathrm{cm}^{2}(a), j=10^{5} \mathrm{~W} / \mathrm{cm}^{2}(b-d)$. Примесные центры не заселены до начала действия импульса.

Скорость двухфотонного перехода „валентная зонапримесь“" определяется выражением [37]

$$
\sigma_{v t}^{(2)}=\frac{2^{11 / 2} e^{4} \pi N_{t}^{2} m_{v}^{3 / 2} \Delta^{1 / 2}}{m^{2} c^{2} \varepsilon_{\infty} \hbar^{6} \omega^{6}}\left|M_{v t}^{(2)}\right|^{2},
$$

где $\Delta=-E_{g}+2 \hbar \omega+\left|E_{t}\right|$, а приведенный составной матричный элемент $M_{v t}^{(2)}$ определяется выражением

$$
\begin{aligned}
& \left|M_{v t}^{(2)}\right|^{2}=\frac{p_{c v}^{4}}{18 m^{2} \hbar^{2} \omega^{2}} \\
& +\left(\frac{4\left(1+\gamma^{-1} / 3\right)^{2}}{3 m_{v}\left(\gamma^{-1} \Delta+\left|E_{t}\right|^{2}\right)}-\frac{2 \Delta}{9 m_{v} \hbar^{2} \omega^{2}}\right) p_{c v}^{2}+\frac{2 m^{2} \Delta^{2}}{5 m_{v}^{2} \hbar^{2} \omega^{2}}
\end{aligned}
$$

В работе [33] было получено выражение для вероятности двухэлектронного перехода, когда в процессе принимают участие два примесных центра:

$\gamma_{v t}^{(1)}\left(n_{c}, j, \widetilde{R}\right)=\frac{2^{9} e^{6} \gamma^{4} m_{\nu}^{7} m^{5} p_{c \nu}^{2} a_{0}^{9} k_{0 v} N_{t}^{4} n_{c} j}{\varepsilon_{\infty}^{1 / 2} \varepsilon_{l}^{2} c \hbar^{15} \omega^{2} \widetilde{R}^{2}}\left|\widetilde{M}_{c v, A B}(\widetilde{R})\right|^{2}$, где безразмерный приведенный матричный элемент процесса $\widetilde{M}_{c v, A B}(\widetilde{R})$ дается выражением

$$
\begin{aligned}
& \widetilde{M}_{c v, A B}(\widetilde{R})=\frac{1}{\gamma a_{1}^{2} a_{2}^{2} c_{1}^{2}}\left(1-\frac{a_{1}^{2} e^{-a_{1} \tilde{R}}-a_{2}^{2} e^{-a_{2} \tilde{R}}}{a_{1}^{2}-a_{2}^{2}}\right) \\
& +\sum_{j=1}^{2} \gamma_{j}\left[\frac{1}{b_{1}^{2} b_{2}^{2} b_{3 j}^{2}}-\frac{e^{-b_{1} \tilde{R}}}{b_{1}^{2}\left(b_{1}^{2}-b_{2}^{2}\right)\left(b_{1}^{2}-b_{3}^{2}\right)}\right. \\
& \left.-\frac{e^{-b_{2} \tilde{R}}}{b_{2}^{2}\left(b_{2}^{2}-b_{1}^{2}\right)\left(b_{2}^{2}-b_{3 j}^{2}\right)}-\frac{e^{-b_{3 j} \tilde{R}}}{b_{3 j}^{2}\left(b_{3 j}^{2}-b_{1}^{2}\right)\left(b_{3 j}^{2}-b_{2}^{2}\right)}\right] .
\end{aligned}
$$

В формуле (7) используются следующие обозначения: $\mu=2 m_{v} a_{0}^{2} / \hbar^{2}, \quad k_{0 v}^{2}=\mu\left(2\left|E_{t}\right|-E_{g}+\hbar \omega\right), \quad a_{1}^{2}=\gamma \mu\left|E_{t}\right|$, $a_{2}^{2}=\mu\left[(2+\gamma)\left|E_{t}\right|-E_{g}+\hbar \omega\right], b_{1}^{2}=\gamma \mu\left(3\left|E_{t}\right|-E_{g}+\hbar \omega\right)$, $b_{2}^{2}=\mu\left|E_{t}\right|, b_{31}^{2}=\gamma \mu\left|E_{t}\right|, b_{32}^{2}=\mu\left(\left|E_{t}\right|+\hbar \omega\right), c_{1}^{2}=\mu\left[2\left|E_{t}\right|+\right.$ $\left.+\gamma^{-1}\left(2\left|E_{t}\right|-E_{g}+\hbar \omega\right)\right], \gamma_{1}=\gamma, \gamma_{2}=1, \varepsilon_{l}-$ продольная диэлектрическая проницаемость, $a_{0}-$ постоянная решетки, $\widetilde{R}$ - отношение расстояния между примесными центрами к $a_{0}$.

Для того, чтобы получить вероятность перехода в единице объема, необходимо проинтегрировать выра- 

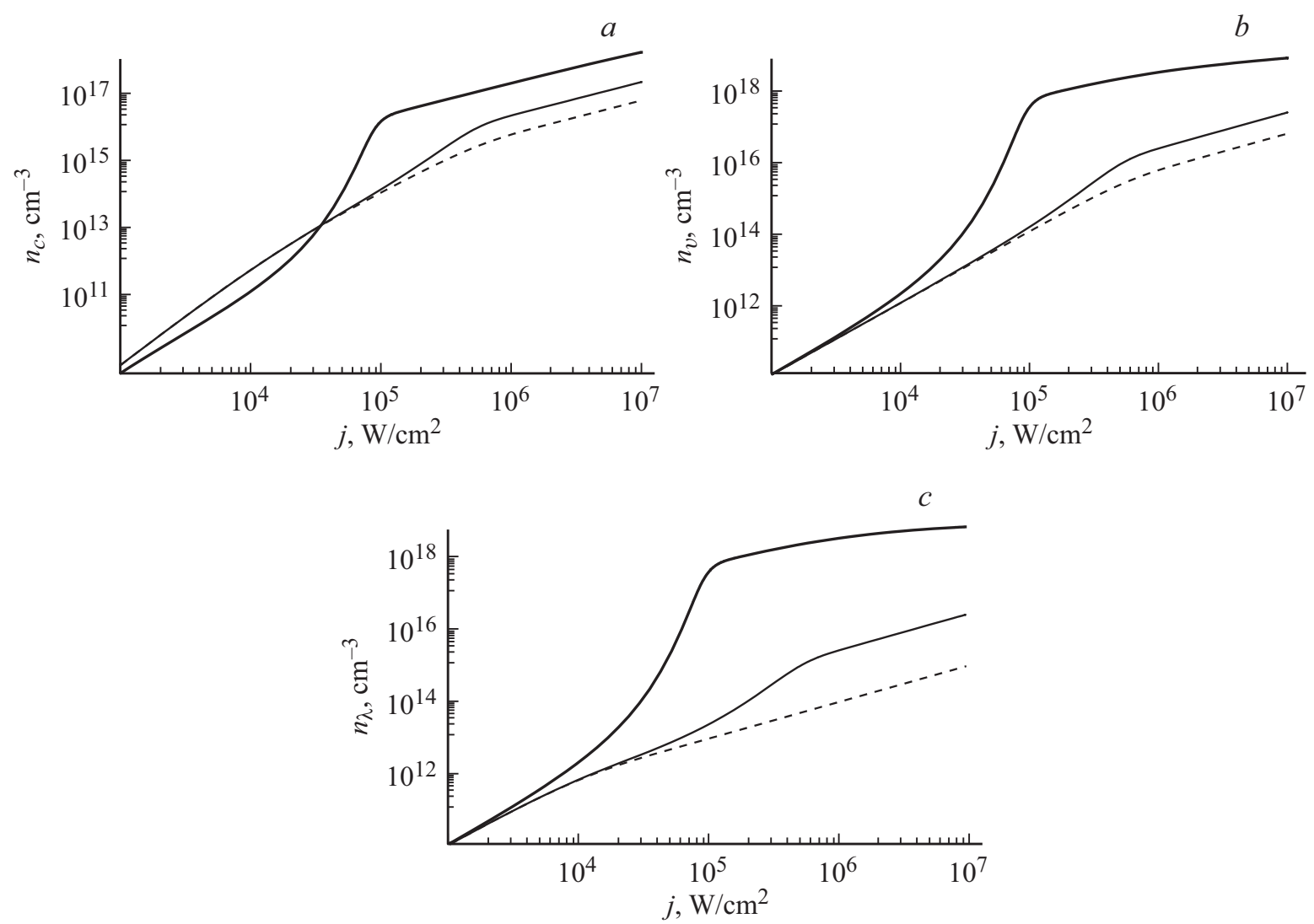

Рис. 3. Зависимости концентраций электронов в зоне проводимости $n_{c}(a)$, дырок в валентной зоне $n_{v}(b)$, электронов на примесных центрах $n_{\lambda}(c)$ от интенсивности излучения $j$. Обозначения кривых такие же, как на рис. 2. Концентрация примесных центров $n_{i}=10^{19} \mathrm{~cm}^{-3}$. Время, прошедшее с момента начала действия излучения, $\tau=10^{-8} \mathrm{~s}$. Примесные центры не заселены до начала действия импульса.

жение (6) с учетом (7) по объему материала. Поскольку при больших значениях $\widetilde{R}$ правая часть (6) пропорциональна $\widetilde{R}^{-2}$, очевидно, что такой интеграл расходится в соответствии с дальнодействующей природой кулоновского взаимодействия. Следует, однако, иметь в виду, что фактически фигурирующие в (6) интегралы перекрывания волновых функций примесных центров необходимо учитывать лишь для расстояний, не превышающих длину когерентности $R_{c}$ для этих функций, причем $R_{c} \sim l$, где $l-$ длина свободного пробега электронов или дырок. Для того, чтобы учесть указанное обстоятельство, введем в подынтегральную функцию экспоненциальный множитель

$$
\gamma_{\nu \lambda}^{(1)}=4 \pi a_{0}^{3} \int_{0}^{\infty} \gamma_{\nu \lambda}^{(1)}\left(n_{c}, j, \widetilde{R}\right) \widetilde{R}^{2} e^{-\tilde{R} / \tilde{R}_{c}} d \widetilde{R},
$$

где $\widetilde{R}_{c}=R_{c} / a_{0}$.

В случае, когда примесные центры распределены не равномерно в объеме полупроводника, а сосредоточены в „кластерах“ со средним линейным размером $R_{0} \sim R_{c}$, с концентрацией примесей в „Кластере“ $\alpha n_{i}(\alpha \gg 1)$, в системе уравнений баланса (1) величина $\gamma_{v t}^{(1)}$ будет определяться не формулой (8), а выражением

$$
\gamma_{\nu \lambda}^{(1)}=4 \pi \alpha a_{0}^{3} \int_{0}^{\tilde{R}_{0}} \gamma_{\nu \lambda}^{(1)}\left(n_{c}, j, \widetilde{R}\right) \widetilde{R}^{2} d \widetilde{R},
$$

где $\alpha n_{i}-$ концентрация примесей в кластере, $R_{0}$ линейный размер кластера, $\widetilde{R}_{0}=R_{0} / a_{0}$.

\section{Результаты расчетов}

На рис. 2 представлены расчетные зависимости концентраций неравновесных фотогенерированных электронов и дырок и заселенностей примесных состояний от времени $t$, прошедшего с момента начала действия лазерного излучения. Расчеты выполнены для случаев, когда действует лишь каскадный двухфотонно-однофотонный механизм возбуждения, либо когда в генерации неравновесных ЭДП участвует также двухцентровый механизм переходов „валентная зона-примесь“. Кроме того, рассматриваются случаи равномерного распределения примесей по объему кристалла и более высокой концентрации примесей в отдельных „кластерах“. Расчеты выполнялись при следующих значениях параметров: $m_{v}=0.7 m, m_{c}=0.4 m, E_{g}=2 \mathrm{eV}, E_{t}=0.7 \mathrm{eV}$, 


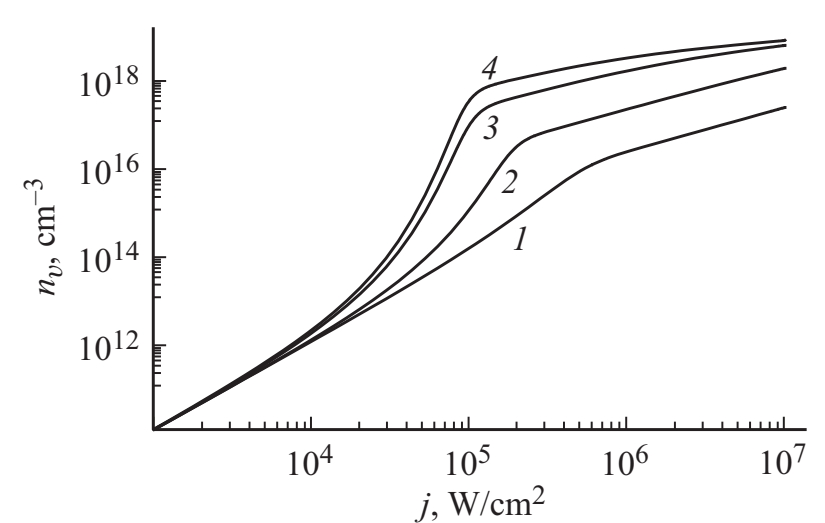

Pис. 4. Зависимости концентраций дырок в валентной зоне $n_{v}$ от интенсивности лазерного излучения для различных „кластеров“. Длительность импульса $\tau=10^{-8}$ s. $R_{0}=1000 a_{0}$. Отношение концентрации примесей в кластере к средней концентрации во всем объеме материала $\alpha=1$ (1), 10 (2), $100(3), 300(4)$.

$p_{c v}=3 \cdot 10^{-19} \mathrm{~g} \cdot \mathrm{cm} / \mathrm{s}, \varepsilon_{l}=3, \varepsilon_{\infty}=3, d=3 \cdot 10^{-8} \mathrm{~cm}^{3} \mathrm{~s}^{-1}$, $\hbar \omega=0.9 \mathrm{eV}, \quad n_{i}=10^{19} \mathrm{~cm}^{-3}, \quad \alpha=300, \quad R_{0}=1000 a_{0}$, $R_{c}=1000 a_{0}, a_{0}=3 \cdot 10^{-8} \mathrm{~cm}$.

Видно, что при концентрации примесей $n_{i} \sim 10^{19} \mathrm{~cm}^{-3}$ учет двухцентровых переходов приводит к значительному увеличению концентрации неравновесных носителей заряда и заполнения примесных состояний. В случае, когда примеси сосредоточены в „кластерах“, этот эффект существенно усиливается. Время установления квазиравновесных концентраций $\tau_{e q}$ обратно пропорционально интенсивности света $j$ и составляет $\tau_{e q} \sim 10 \mathrm{~ns}$ при $j=10^{5} \mathrm{~W} / \mathrm{cm}^{2}$. Следует отметить, что при рассмотренном двухцентровом механизме возбуждения кристалла бо́льшая часть покинувших валентную зону электронов заполняет примесные состояния, так что концентрация фотогенерированных дырок в валентной зоне значительно превышает концентрацию свободных электронов в зоне проводимости.

На рис. 3 представлены зависимости концентраций неравновесных носителей от интенсивности индуцирующего излучения в зоне проводимости и валентной зоне и концентрации электронов в примесных состояниях от интенсивности излучения. Видно, что для принятых в расчетах значений параметров кристалла действие двухцентрового механизма возбуждения приводит к появлению области интенсивностей $j$, в которой концентрации фотовозбужденных электронов и дырок очень резко возрастают с ростом $j$, что как раз характерно для процессов типа ФЛ.

На рис. 4 приведена зависимость концентрации дырок в валентной зоне от интенсивности лазерного излучения для различных параметров „кластеров“. Как и следовало ожидать, увеличение концентрации примесей в „кластере“ при неизменном размере кластера приводит к увеличению концентрации неравновесных дырок.
Укажем примеры примесей, положение уровней которых в запрещенной зоне кристалла позволяет реализовать предложенный в работе механизм фотовозбуждения. Положение примесного уровня и энергия кванта падающего излучения должны удовлетворять следующим условиям: $\hbar \omega>\left|E_{t}\right|$ и $E_{g}-\left|E_{t}\right|>\hbar \omega>$ $>\left(E_{g}-\left|E_{t}\right|\right) / 2$. Таким условиям удовлетворяют, например, примесь хрома в $\mathrm{GaAs}\left(E_{g}=1.42 \mathrm{eV}\right.$, $\left.\left|E_{t}\right|=0.63 \mathrm{eV}, \hbar \omega \approx 0.64-0.67 \mathrm{eV}\right)$ [38], примесь меди B InP $\left.E_{g}=1.34 \mathrm{eV},\left|E_{t}\right|=0.66 \mathrm{eV}, \hbar \omega \approx 0.66 \mathrm{eV}[39]\right)$, примесь кремния в $\mathrm{GaN}\left(E_{g}=3.2 \mathrm{eV},\left|E_{t}\right|=0.59 \mathrm{eV}\right.$, $\hbar \omega \approx 1.4-1.5 \mathrm{eV})[40]$.

\section{Заключение}

Как показывают результаты расчетов, учет двухцентрового механизма фотовозбуждения прозрачных кристаллов с глубокими примесями приводит к отчетливо выраженному пороговому характеру процесса каскадной генерации неравновесных электронов и дырок. Фактически предлагаемый механизм позволяет реализовать пороговое переключение материала из непроводящего в проводящее состояние при достаточно умеренных интенсивностях света порядка $10^{5} \mathrm{~W} / \mathrm{cm}^{2}$, при этом концентрация дырок в валентной зоне оказывается на несколько порядков выше, чем при учете лишь двухфотонно-однофотонного каскадного механизма возбуждения. Увеличение интенсивности возбуждающего лазерного излучения приводит к увеличению квазиравновесных концентраций неравновесных носителей заряда и к уменьшению времени переключения материала, при этом время установления квазиравновесных концентраций $\tau_{e q} \propto j^{-1}$.

Укажем ряд аспектов рассмотренной задачи, которые предстоит исследовать более детально. Так, в частности, при высоких интенсивностях света $j$ состояния вблизи дна зоны проводимости $c$ оказываются заполненными, а состояния вблизи потолка валентной зоны $v$ опустошенными (т.е. возникает высокая концентрация дырок). Это обстоятельство может оказаться существенным в случае, когда точка двухфотонного резонанса на переходе „валентная зона $v$-примесь“ расположена в $\mathbf{k}$-пространстве в непосредственной близости от потолка зоны $v$, либо когда точка однофотонного резонанса на переходе „примесь-зона проводимости $c^{\text {“ }}$ находится вблизи дна зоны $c$. Влияние этих факторов на скорости переходов может быть учтено аналогично тому, как это сделано в работах [24,27,30-32]. Очевидно также, что при высокой концентрации примесных центров возможно образование примесной зоны. В этом случае возникает ситуация, промежуточная между теми, что рассмотрены в [32-34] и в настоящей работе. Для исследования такой задачи необходим выход за рамки модели потенциала нулевого радиуса. Кроме того, в дальнейшем может быть учтено влияние заполнения примесных центров на скорость рекомбинации электронов и дырок. 
Вероятно, учет этого фактора привел бы к усилению полученного эффекта.

Работа выполнена при государственной финансовой поддержке ведущих университетов Российской Федерации (субсидия 074-U01), базовой части государственного задания в сфере научной деятельности - проект 5.4681.2017/6.7 и РФФИ (грант 16-32-00102).

\section{Список литературы}

[1] Chivian J.S., Case W.E., Eden D.D. // Appl. Phys. Lett. 1979. V. 35. N 2. P. 124. doi 10.1063/1.91044

[2] Kueny A.W., Case W.E., Koch M.E. // JOSA B. 1989. V. 6. N 4. P. 639. doi 10.1364/JOSAB.6.000639

[3] Case W.E., Koch M.E., Kueny A.W. // J. Luminesc. 1990. V. 45. P. 351. doi 10.1016/0022-2313(90)90191-D

[4] Ni H., Rand S.C. // Opt. Lett. 1991. V. 16. N 18. P. 1424. doi 10.1364/OL.16.001424

[5] Kueny A.W., Case W.E., Koch M.E. // JOSA B. 1993. V. 10. N 10. P. 1834. doi 10.1364/JOSAB. 10.001834

[6] Chen Y., Auzel F. // J. Phys. D: Appl. Phys. 1995. V. 28. N 1. P. 207. doi $10.1088 / 0022-3727 / 28 / 1 / 029$

[7] Auzel F., Chen Y. // J. Luminesc. 1995. V. 65. N 1. P. 45. doi 10.1016/0022-2313(94)00071-J

[8] Auzel F., Chen Y.H. // J. Non-Crystalline Solids. 1995. V. 184. P. 57. doi 10.1016/0022-3093(94)00595-8

[9] Goldner Ph., Pelle F. // Opt. Materials. 1996. V. 5. N 4. P. 239. doi 10.1016/0925-3467(96)00003-1

[10] Auzel F. // Acta Physica Polonica A. 1996. V. 90. N 1. P. 7. doi 10.12693/APhysPolA.90.7

[11] Pellé F., Goldner P. // Acta Physica Polonica A. 1996. V. 90. N 1. P. 197. doi 10.12693/APhysPolA.90.197

[12] Guy S., Joubert M.-F., Jacquier B. // Phys. Rev. B. 1997. V. 55. N 13. P. 8240. doi 10.1103/PhysRevB.55.8240

[13] Joubert M.-F. // Opt. Materials. 1999. V. 11. P. 181. doi 10.1016/S0925-3467(98)00043-3

[14] Gatch D.B., Dennis W.M., Yen W.M. // Phys. Rev. B. 2000. V. 62. N 16. P. 10790. doi 10.1103/PhysRevB.62.10790

[15] Hehlen M.P., Kuditcher A., Lenef A.L., Ni H., Shu Q., Rand S.C., Rai J., Rai S. // Phys. Rev. B. 2000. V. 61. N 2. P. 1116-1124. doi 10.1103/PhysRevB.61.1116

[16] Перлин E.Ю., Ткачук A.M., Joubert M.-F., Moncorge R. // Опт. и спектр. 2001. Т. 90. № 5. С. 772; Perlin E.Yu., Tkachuk A.M., Joubert M.-F., Moncorge R. // Opt. Spectrosc. 2001. V. 90. N 5. P. 691. doi 10.1134/1.1374657.

[17] Singh A.K., Kumar K., Pandey A.C., Parkash O., Rai S.B., Kumar D. // Appl. Phys. B. 2011. V. 104. P. 1035. doi 10.1007/s00340-011-4673-2

[18] Verma R.K., Singh S.K., Rai S.B. // Current Applied Physics. 2012. V. 12. N 6. P. 1481. doi 10.1016/j.cap.2012.04.018

[19] Babu P., Martín I.R., Krishnaiah K.V., Seo H.J., Venkatramu V., Jayasankar C.K., Lavín V. // Chem. Phys. Lett. 2014. V. 600. P. 34. doi 10.1016/j.cplett.2014.03.048

[20] Rathaiah M., Martín I.R., Babu P., Lingannaa K., Jayasankar C.K., Lavín V., Venkatramu V. // Opt. Materials. 2015. V. 39. N 1. P. 16. doi 10.1016/j.optmat.2014.10.050
[21] Перлин Е.Ю. // Опт. и спектр. 2001. Т. 91. № 5. С. 777; Perlin E.Yu. // Opt. Spectrosc. 2001. V. 91. N 5. P. 729 doi 10.1134/1.1420854

[22] Perlin E.Yu. // J. Luminesc. 2001. V. 94-95. P. 249. doi 10.1016/S0022-2313(01)00288-5

[23] Перлин Е.Ю., Иванов А.В., Левицкий Р.С. // ЖЭТФ. 2003. T. 123. № 3. C. 612; Perlin E.Yu., Ivanov A.V., Levitskií R.S. // JETP. 2003. V. 96. N 3. P. 543. doi 10.1134/1.1567429

[24] Перлин Е.Ю., Иванов А.В., Левицкий Р.С. // ЖЭТФ. 2005. T. 128. № 2. C. 411; Perlin E.Yu., Ivanov A.V., Levitskǐ R.S. // JETP. 2005. V. 101. N 2. P. 357. doi 10.1134/1.2047802

[25] Перлин Е.Ю., Левицкий Р.С. // Оптич. журн. 2006. Т. 73. № 1. C. 3; Perlin E.Yu., Levitskii R.S. // J. Opt. Technol. 2006. V. 73. N 1. P. 1. doi 10.1364/JOT.73.000001

[26] Перлин Е.Ю., Иванов А.В., Левицкий Р.С. // Оптич. журн. 2006. Т. 73. № 1. С. 12; Perlin E.Yu., Ivanov A.V., Levitskii R.S. // J. Opt. Technol. 2006. V. 73. N 1. P. 9. doi 10.1364/JOT.73.000009

[27] Левицкий Р.С., Иванов А.В., Перлин Е.Ю. // Оптич. журн. 2006. T. 73. № 2. C. 3; Levitskii R.S., Ivanov A.V., Perlin E.Yu. // J. Opt. Technol. 2006. V. 73. N 2. P. 71. doi 10.1364/JOT.73.000071

[28] Перлин Е.Ю., Левицкий Р.С. // Опт. и спектр. 2007. Т. 102. № 2. C. 303; Perlin E.Yu., Levitskii R.S. // Opt. Spectrosc. 2007. V. 102. N 2. P. 262. doi 10.1134/S0030400X07020154

[29] Иванов А.В., Левицкий Р.С., Перлин Е.Ю. // Опт. и спектр. 2009. T. 107. № 2. C. 272; Ivanov A.V., Levitskii R.S., Perlin E.Yu. // Opt. Spectrosc. 2009. V. 107. N 2. P. 255. doi 10.1134/S0030400X09080153

[30] Перлин Е.Ю., Иванов А.В., Попов А.А. // Опт. и спектр. 2012. T. 113. № 4. C. 418; Perlin E.Yu., Ivanov A.V., Popov A.A. // Opt. Spectrosc. 2012. V. 113. N 4. P. 376. doi 10.1134/S0030400X12100074

[31] Перлин Е.Ю., Иванов А.В., Попов А.А. // Опт. и спектр. 2012. T. 113. № 4. C. 426; Perlin E.Yu., Ivanov A.V., Popov A.A. // Opt. Spectrosc. 2012. V. 113. N 4. P. 383. doi 10.1134/S0030400X12100086

[32] Перлин Е.Ю., Иванов А.В., Попов А.А. // Опт. и спектр. 2013. T. 115. № 5. C. 830; Perlin E.Yu., Ivanov A.V., Popov A.A. // Opt. Spectrosc. 2013. V. 115. N 5. P. 739. doi 10.1134/S0030400X13110179

[33] Перлин Е.Ю., Левицкий Р.С., Иванов А.В., Елисеев К.A. // Опт. и спектр. 2015. Т. 117. № 2. С. 242. doi 10.7868/S0030403415020154; Perlin E.Yu., Levitskii R.S., Ivanov A.V., Eliseev K.A. // Opt. Spectrosc. 2015. V. 118. N 2. P. 229. doi $10.1134 / \mathrm{S} 0030400 \mathrm{X} 15020150$

[34] Перлин Е.Ю., Левицкий Р.С., Иванов А.В., Елисеев К.А. // Опт. и спектр. 2015. Т. 119. № 2. С. 226.

http://dx.doi.org/10.7868/S0030403415080188;

Perlin E.Yu., Levitskii R.S., Ivanov A.V., Eliseev K.A. // Opt. Spectrosc. 2015. V. 119. N 2. P. 216. http://dx.doi.org/10.1134/S0030400X15080184

[35] Lucovsky G. // Solid State Commun. 1965. V. 3. N 9. P. 299. doi 10.1016/0038-1098(65)90039-6

[36] Бонч-Бруевич В.Л. // Вестн. Московского университета. Сер. физика и астрономия. 1971. Т. 12. № 5. С. 586. 
[37] Левицкий Р.С., Перлин Е.Ю., Попов А.А. // Оптич. журн. 2010. T. 77. № 10. C. 3; Levitski R.S., Perlin E.Yu., Popov A.A. // J. Opt. Technol. 2010. V. 77. N 10. P. 77. doi 10.1364/JOT.77.000593

[38] Milnes A.G. Deep Impurities in Semiconductors. John Wiley \& Sons Inc, 1973. 544 р.; Милнс А. Примеси с глубокими уровнями в полупроводниках. М.: Мир, 1977. 562 с.

[39] Levinshtein M., Rumyantsev S., Shur M. Handbook Series on Semiconductor Parameters. V. 1. World Scientific Pub. Co Inc, 1996. $218 \mathrm{p}$.

[40] Hacke P., Maekawa A., Koide N., Hiramatsu K., Sawaki N. // Jpn. J. Appl. Phys. 1994. V. 33. Pt. 1. N 12A. P. 6443 doi 10.1143/JJAP.33.6443 